\title{
CERTAIN FOURIER TRANSFORMS OF DISTRIBUTIONS
}

\author{
KINSAKU TAKANO
}

(Received June 25, 1951)

\section{Introduction and summary}

Recently E. Lukacs and O.Szász [3] gave a necessary condition which the reciprocal of a polynomial without multiple roots must satisfy in order to be a charcteristic function. The assumption that the polynomial has no multiple roots is, however, unnecessary, and moreover, if the degree of the polynomial is less than 4 , the condition is not only necessary but also sufficient. The condition is not sufficient in case when the degree of the polynomial is equal to 5 . The proof and example will be given in $\S 3$. The principle of the proof is the same as one given by E. Lukacs and O. Szász.

Let $L$ be the probability law defined by the density function

$$
f(x)= \begin{cases}\alpha e^{-\alpha x}, & \text { if } x \geqq 0, \\ 0, & \text { if } x<0,\end{cases}
$$

where $\alpha$ is positive, then the characteristic function of $L$ is given by

$$
\phi(t)=\left(1-\frac{i t}{\alpha}\right)^{-1} .
$$

The probability law $L$ has a curious property; starting from the law $L$, one obtains the same symmetric law by $X=X_{1}-X_{2}$, where $X_{1}$ and $X_{2}$ are independent random variables with the law $L$, or by $X=\varepsilon X_{1}$, where $\varepsilon$ and $X_{1}$ are independent random variables, $\varepsilon$ taking on \pm 1 with equal probabilities, the law of $X_{1}$ being $L$. The identity of the two laws, which are in general different, is expressed by the formula

$$
\frac{1}{2}[\varphi(t)+\varphi(-t)]=\varphi(t) \varphi(-t) \text {. }
$$

Under the condition that $\varphi(t)$ is a non-vanishing characteristic function, the solution of (1.1) is given by

$$
\varphi(t)=\frac{1}{1+i \omega(t)},
$$

where $\omega(t)$ is a real valued function of $t$ such that

$$
\omega(-t)=-\omega(t) \text {. }
$$

A question arises, if there is another characteristic function of this form than those obtained by putting $\omega(t)=c t$. P. Lévy [2] expressed himself that this question did not seem to have been solved. We will give an answer to this problem in the affirmative in $\S 4$.

Professor T. Kawata has informed me the result of E. Lukacs and O. Szász [3], which has not been published as yet, and I have greatly profited from his valuable remarks. I wish to express my sincere thanks to him. 


\section{Auxiliary formulas and lemmas}

Formulas. (See [3] §3).

If $\Re(\alpha)>0$, that is, $\alpha$ is a real positive number or a complex number with positive real part, and $\lambda$ is a positive integer,

$$
\frac{1}{2 \pi} \int_{-\infty}^{\infty} \frac{e^{-i t x}}{\left(1-\frac{i t}{\alpha}\right)^{\lambda}} d t= \begin{cases}\frac{\alpha^{\lambda}}{\Gamma(\lambda)} x^{\lambda-1} e^{-\alpha x}, & \text { if } x>0 \\ 0, & \text { if } x<0\end{cases}
$$

and

$$
\int_{0}^{\infty} \frac{\alpha^{\lambda}}{\Gamma(\lambda)} x^{\lambda-1} e^{-\alpha x} e^{i t x} d x=\left(1-\frac{i t}{\alpha}\right)^{-\lambda}
$$

If $\Re(\beta)>0$ and $\lambda$ is a positive integer,

and

$$
\frac{1}{2 \pi} \int_{-\infty}^{\infty} \frac{e^{-i t x}}{\left(1+\frac{i t}{\beta}\right)^{\lambda}} d t= \begin{cases}\frac{\beta^{\lambda}}{\Gamma(\lambda)}(-x)^{\lambda-1} e^{\beta x}, & \text { if } x<0, \\ 0, & \text { if } x>0,\end{cases}
$$

$\left.\int_{-\infty} \Gamma(\lambda)(-x)\right)^{\lambda-1} e^{\beta} e^{\operatorname{ma} d x}=\left(1+\frac{\pi}{\beta}\right)$.

In (2.1) and (2.3), if $\lambda=1, \int_{-\infty}^{\infty}$ means $\lim _{\tau \rightarrow \infty} \int_{-\tau}^{\tau}$.

LEMMA 1. (well-known). If a non-negative real valued almost periodic function $g(x)$ satisfies

$$
M(g(x))=\lim _{\tau \rightarrow \infty} \frac{1}{2 \tau} \int_{-\tau}^{\tau} g(x) d x=0
$$

then $g(x) \equiv 0$.

LEMMA 2. Let

$$
g(x)=\sum_{j=1}^{m}\left(B_{j} e^{-i b j x}+\bar{B}_{j} e^{i b j x}\right)
$$

where $0<b_{1}<b_{2}<\cdots<b_{m}, \quad B_{j} \neq 0(j=1,2, \cdots m)$, and $\bar{B}$ expresses the conjugate complex number of a complex number $B$, then

$$
\liminf _{x \rightarrow \infty} g(x)<0, \quad \liminf _{x \rightarrow-\infty} g(x)<0 .
$$

Proof. It is evident that

(2.6) $g(x)$ is a real valued almost periodic function,

(2.7) $\quad M(g(x))=0$, and

$$
M\left(|g(x)|^{2}\right)=2 \sum_{j=1}^{m}\left|B_{j}\right|^{2}>0 .
$$


If $g(x)$ is non-negative, from Lemma 1 and (2.7), it follows that $g(x) \equiv 0$ which contradicts (2.8). Therefore there exists at least one value $x_{0}$ such that $g\left(x_{0}\right)<0$. Using (2.6), we have

$$
\liminf _{x \rightarrow \infty} g(x)<0, \quad \liminf _{x \rightarrow-\infty} g(x)<0 .
$$

3. TheOREM 3.1. (Eugene Lukacs and Otto Szász). In order that the complex valued function of a real variable $t$

(3. 1) $\varphi(t)=\left\{c_{0}+c_{1}(i t)+c_{2}(i t)^{2}+\cdots+c_{N}(i t)^{N}\right\}^{-1},\left(i=\sqrt{-1}, c_{N} \neq 0\right)$, be the characteristic function of a probability distribution, it is necessary that (3.2) $c_{0}=1$ and all $c_{j}$ are real,

(3.3) the polynomial with real coefficients

$$
Q(z)=1+c_{3} z+c_{2} z^{2}+\cdots+c_{N} z^{N}
$$

has no pure imaginary roots, and that

(3.4) if $a \pm i b(a \neq 0, b \neq 0)$ is a pair of complex roots of the polynomial $Q(z)$ ther it has at least one real root $c$ such that $\operatorname{sign} c=\operatorname{sign} a$ and $|c| \leqq|a|$.

PROOF. If the function $\varphi(t)$ is the characteristic function of a destribution then :

$$
\begin{aligned}
& \varphi(0)=1, \\
& |\varphi(t)| \leqq 1, \\
& \varphi(-t)=\overline{\varphi(t)} .
\end{aligned}
$$

From (3.5), we have $c_{0}=1$. From (3.7), we have

$$
\begin{aligned}
& 1+c_{1}(-i t)+c_{2}(-i t)^{2}+\cdots+c_{N}(-i t)^{N} \\
= & 1+\bar{c}_{1}(-i t)+\bar{c}_{2}(-i t)^{2}+\cdots+\bar{c}_{N}(-i t)^{N}
\end{aligned}
$$

for all real $t$. Therefore all $c_{j}$ must be real. (3.3) is derived from (3.6). In the sequel (3.2) and (3.3) are considered to be satisfied.

Let the zeros of the polynomial $Q(z)$ be

$$
z=\alpha_{j}(j=1,2, \cdots, m) \quad \text { and } \quad z=-\beta_{k} \quad(k=1,2, \cdots, n)
$$

where $\mathfrak{R}\left(\boldsymbol{\alpha}_{j}\right)>0, \mathfrak{R}\left(\boldsymbol{\beta}_{k}\right)>0$, and let their multiplicities be $p_{j}$ and $q_{k}$. If $\alpha_{j}$ is not real, there exists $\alpha_{j^{\prime}}$, which is conjugate complex with $\alpha_{j}$ and $p_{j}=p_{j^{\prime}}$. The same is true for $\beta_{k}^{\prime}$ 's and $\boldsymbol{q}_{k}$ 's.

The function (3.1) can then be written

$$
\varphi(t)=\left[\prod_{j=1}^{m}\left(1-\frac{i t}{\alpha_{j}}\right)^{p_{j}} \prod_{k=1}^{n}\left(1+\frac{i t}{\beta_{k}^{-}}\right)^{q_{k}}\right]^{-1} .
$$

If $\varphi(t)$ is decomposed into partial fractions, it is seen that

$$
\varphi(t)=\sum_{j=1}^{m} \sum_{p=1}^{p_{j}} \frac{A_{j p}}{\left(1-\frac{i t}{\alpha_{j}}\right)^{p}}+\sum_{k=1}^{n} \sum_{q=1}^{q_{k}} \frac{B_{k q q}}{\left(1+\frac{i t}{\beta_{k}}\right)^{q}}
$$

where

(3.10) if $\alpha_{j}$ is real then $A_{j p}$ is real, and if $\alpha_{j}=\overline{\alpha_{j^{\prime}}}$, then $A_{j p}=\bar{A}_{j^{\prime} p}$ and the same is true for $\beta_{k}$ 's and $B_{k y}^{\prime}$ s.

If $m=0$, i. e., if there are no roots with positive real part the first term 
of (3.9) is omitted, and if $n=0$, the second term.

Let

$$
f(x)=\frac{1}{2 \pi} \int_{-\infty}^{\infty} e^{-i t x} \varphi(t) d t,
$$

then, we have from (2.1)-(2.4),

$$
f(x)= \begin{cases}\sum_{j=1}^{m} \sum_{p=1}^{p_{j}} \frac{A_{j p} \alpha_{i}^{p}}{\Gamma(p)} x^{p-1} e^{-\alpha_{i} x}, & (x>0), \\ \sum_{k=1}^{n} \sum_{i=1}^{n} \frac{B_{k \neq} \beta_{k}^{q}}{\Gamma(q)}(-x)^{q-1} e^{\beta_{k} x}, & (x<0),\end{cases}
$$

and

$$
\varphi(t)=\int_{-\infty}^{\infty} e^{i t x} f(x) d x, \quad \int_{-\infty}^{\infty} f(x) d x=\varphi(0)=1 .
$$

Using (3.10), $f(x)$ is seen to be real.

Since

$$
|\varphi(t)| \sim\left|c_{N} t^{N}\right|^{-1}, \quad(t \rightarrow \pm \infty)
$$

it follows that $|\varphi(t)|$ is integrable over $(-\infty, \infty)$, assuming that $N \geqq 2$. Therefore, if $\varphi(t)$ is a characteristic function, then $f(x)$ must be the probability density corresponding to $\phi(t)$ (Cramér [1], p. 94). Since $f(x)$ is continuous, it is necessary that $f(x) \geqq 0$ for every $x$ in order that $f(x)$ is a probability density.

Conversely, if $f(x) \geqq 0$, from (3.13) it follows that $\varphi(t)$ is a characteristic function (This fact is used for the proof of Theorem 3.2).

To derive the condition (3.4), it is sufficient to prove that if none of $\alpha$, corresponding to the smallest $\Re\left(\alpha_{j}\right)$ are real, then there exists at least one value $x$ such that $f(x)<0$.

Let

$$
\alpha_{j}=a_{j}+i b_{j} \quad\left(a_{j}, b_{j} \text { real }\right) \quad(j=1,2, \cdots, m) .
$$

We may assume that

We can find $m_{0}$ such that either

$$
a_{1} \leqq a_{i:} \leqq \cdots \leqq a_{m} .
$$

$$
\begin{aligned}
& a_{1}=a_{2}=\cdots=a_{m_{0}}<a_{i i_{0}+1} \leqq \cdots \leqq a_{m,} \text { or } \\
& m_{0}=m \text { and } \boldsymbol{a}_{1}=\boldsymbol{a}_{2}=\cdots=\boldsymbol{a}_{m} .
\end{aligned}
$$

Let

$$
\begin{aligned}
& \max \left(p_{1}, p_{2}, \cdots, p_{m_{0}}\right)=s, \\
& \max \left(p_{m_{0}+1}, \cdots, p_{m}\right)=t,
\end{aligned}
$$

then the function (3.12) can be written

$$
f(x)=x^{s-1} e^{-a x} g(x)+O\left(x^{s-2} e^{-a x}\right)+O\left(x^{t-1} e^{-a_{m_{0}+1} x}\right)
$$$$
\text { as } x \rightarrow+\infty \text {, where }
$$

$$
a=a_{1}<a_{m_{0}+1}
$$




$$
g(x)=\sum_{1 \leqq j \leqq m_{0}, p_{j}=s} \frac{A_{j s} \alpha_{i}^{s}}{\Gamma(s)} e^{-i b \rho^{x}} .
$$

If $s=1$ the second term of (3.15) is omitted, and if $m_{0}=m$, the third term.

Because of (3.15) and (3.16), we have

$$
f(x)=x^{s-1} e^{-a x}(g(x)+o(1)), \quad(x \rightarrow \infty) .
$$

We may assume that $b_{j}$ 's which appear in the right hand side of (3.17) and are positive are arranged as

(3.17) can then be written

$$
0<b_{1}<b_{2}<\cdots<b_{m_{1}}
$$

$$
g(x)=\sum_{j=1}^{m_{1}}\left(B_{j} e^{-i \partial j x}+\bar{B}_{j} e^{i \rho_{j} x}\right)
$$

with $B_{j} \neq 0\left(j=1, \cdots m_{1}\right)$. From Lemma 2 , it follows that

$$
\liminf _{x \rightarrow+\infty} g(x)<0 \text {. }
$$

From (3.18) and (3.19), it follows that there exists at least one value $x$ such that $f(x)<0$.

Q.E.D.

COROLLARY. If the polynomial with real coefficients

$$
Q(z)=1+a_{1} z+a_{3} z^{3}+\cdots+a_{n-2} z^{n-2}+a_{n} z^{n}
$$

of degree $n$ without term of degree $n-1$ has only one value of real roots, then $1 / Q(i t)$ cannot be a characteristic function.

PRoof. If $Q(z)$ is decomposed into real factors it is seen that

$$
Q(z)=a_{n}(z-\alpha)^{k} \prod_{i=1}^{l}\left(z^{2}-2 \beta_{j} z+\gamma_{j}\right), \quad(k+2 l=n) .
$$

As the coefficient of $z^{n-1}$ in the right hand side is equal to zero,

$$
-2 \sum_{j=1}^{l} \beta_{j}-k \alpha=0, \quad \text { or, } \quad 2 \sum_{i=1}^{l} \alpha \beta_{j}=-k \alpha^{2}<0 .
$$

Therefore, there exists at least one $j$ such that

$$
\alpha \beta_{j}<0 \text {. }
$$

However, $\beta_{j}$ is the real part of a complex root of $Q(z)$ and $\alpha$ is the only one value of real roots of $Q(z)$. Therefore $Q(z)$ does not satisfy (3.4).

Q. E. D.

THEOREM 3.2. If the polynomial of third degree with real coefficients

$$
Q(z)=1+a_{1} z+a_{2} z^{2}+a_{3} z^{3}
$$

has a real root $\alpha$ and complex roots $\beta \pm i \gamma$, and if

then

$$
\operatorname{sign} \alpha=\operatorname{sign} \beta \text { and }|\alpha| \leqq|\beta| \text {, }
$$

is a characteristic function.

$$
\phi(t)=\frac{1}{Q(i t)}
$$

PRoof. Since, if $\phi(t)$ is a characteristic function, $\varphi(-t)$ is also one, we may assume that $0<\alpha \leqq \beta$ and $\gamma>0$. If $f(x)$ is defined by (3.11), then 
we have (3.13). Therefore, it is sufficient to prove that $f(x) \geqq 0$.

If

$$
\varphi(t)=\left[\left(1-\frac{i t}{\alpha}\right)\left(1-\frac{i t}{\beta+i \gamma}\right)\left(1-\frac{i t}{\beta-i \gamma}\right)\right]^{-1}
$$

is decomposed into partial fractions it is seen that

$$
\varphi(t)=a /\left(1-\frac{i t}{\alpha}\right)+b /\left(1-\frac{i t}{\beta+i \gamma}\right)+\bar{b} /\left(1-\frac{i t}{\beta-i \gamma}\right),
$$

where

$$
a=\left[\left(1-\frac{\alpha}{\beta+i \gamma}\right)\left(1-\frac{\alpha}{\beta-i \gamma}\right)\right]^{-1}=\frac{\beta^{2}+\gamma^{2}}{(\beta-\alpha)^{2}+\gamma^{2}}>0,
$$

and

$$
b=\left[\left(1-\frac{\beta+i \gamma}{\alpha}\right)\left(1-\frac{\beta+i \gamma}{\beta-i \gamma}\right)\right]^{-1}
$$

We have

$$
\begin{aligned}
& f(x)= \frac{1}{2 \pi} \int_{-\infty}^{\infty} \varphi(t) e^{-i t x} d t \\
&= \lim _{\tau \rightarrow \infty} \frac{1}{2 \pi} \int_{-\tau}^{\tau}\left[a e^{-i x t}\left(1-\frac{i t}{\alpha}\right)^{-1}+b e^{-i x t}\left(1-\frac{i t}{\beta+i \gamma}\right)^{-1}\right. \\
&\left.+b e^{-i x t}\left(1-\frac{i t}{\beta-i \gamma}\right)^{-1}\right] d t \\
&= \begin{cases}a \alpha e^{-\alpha x}+b(\beta+i \gamma) e^{-(\beta+i \gamma) x}+\bar{b}(\beta-i \gamma) e^{-(\beta-i \gamma) x} & (x>0), \\
0 & (x<0),\end{cases}
\end{aligned}
$$

If $x>0$,

$$
\begin{aligned}
f(x) & =a \alpha e^{-\alpha x}+2 \Re\left\{b(\beta+i \gamma) e^{-(\beta+i \gamma) x}\right\} \\
& =a \alpha e^{-\alpha x}\left[1-e^{-(\beta-\alpha) x}\left\{\cos \gamma x+\frac{\beta-\alpha}{\gamma} \sin \gamma x\right\}\right] \\
& \geqq 0
\end{aligned}
$$

where we have used that

$$
b(\beta+i \gamma)=-\frac{a \alpha}{2}\left\{1+i \frac{\beta-\alpha}{\gamma}\right\} .
$$

CoROllaRy 1. If a polynomial of degree of $3 n+m(n=1,2, \cdots ; m=0$, $2, \cdots)$ with real coefficients

$$
Q(z)=1+a_{1} z+a_{2} z^{2}+\cdots+a_{3 n+m} z^{3 n+m}
$$

has $n+m$ real roots $\alpha_{j}(j=1,2, \cdots, n+m)$ and $n$ pairs of conjugate complex roots $\beta_{j} \pm i \gamma_{j}(j=1,2, \cdots, n)$ (multiple roots being enumerated by its multiplicity) and if

$$
\alpha_{j} \beta_{j}>0, \quad\left|\alpha_{j}\right| \leqq\left|\beta_{j}\right| \quad(j=1,2, \cdots, n),
$$

then, $1 / Q$ (it) is a characteristic function. 
COROLLARY 2. Under the condition that

$n \leqq 4$

in order that (3.1) be a characteristic function, it is necessary and sufficient that (3.2)-(3.4) holds.

Notice : If $n=5,(3.2)-(3.4)$ is not sufficient in order that (3.1) be a characteristic function.

Example. Let

$$
\varphi(t)=\left[\left(1-\frac{i t}{\alpha}\right)\left(1-\frac{i t}{\beta+i \gamma_{1}}\right)\left(1-\frac{i t}{\beta-i \gamma_{1}}\right)\left(1-\frac{i t}{\beta+i \gamma_{2}}\right)\left(1-\frac{i t}{\beta-i \gamma_{2}}\right)\right]^{-1}
$$

where $\quad 0<\alpha<\beta, \gamma_{1}=\left(2 n+\frac{1}{2}\right) \pi-\theta, \gamma_{2}=\left(2 n+\frac{1}{2}\right) \pi+\theta$,

$$
0<\theta<\frac{\pi}{2} \quad \text { and } n \pi \frac{\sin \theta}{\theta}>1 .(n=1,2,3, \cdots) \text {. }
$$

If $\beta-\alpha$ is sufficiently small, then $\varphi(t)$ cannot be a characteristic function. In this case, $(3.12)$ can be written

$$
f(x)= \begin{cases}A \alpha e^{-\alpha x}[1-g(x ; \beta-\alpha)], & (x>0), \\ 0, & (x<0),\end{cases}
$$

where

Since

$$
\begin{aligned}
A= & \left|\left(1-\frac{\alpha}{\beta+i \gamma_{1}}\right)\left(1-\frac{\alpha}{\beta+i \gamma_{2}}\right)\right|^{-2}>0 \\
g(x ; \varepsilon)= & \frac{e^{-\varepsilon x}}{\gamma_{2}^{2}-\gamma_{1}^{2}}\left\{\left(\varepsilon^{2}+\gamma_{2}^{2}\right)\left(\cos \gamma_{1} x+\frac{\varepsilon}{\gamma_{1}} \sin \gamma_{1} x\right)\right. \\
& \left.-\left(\varepsilon^{2}+\gamma_{1}^{2}\right)\left(\cos \gamma_{2} x+\frac{\varepsilon}{\gamma_{2}} \sin \gamma_{2} x\right)\right\} .
\end{aligned}
$$

$$
\lim _{\varepsilon \rightarrow 0} g(1 ; \varepsilon)=g(1,0)=\frac{\gamma_{2}^{2} \cos \gamma_{1}-\gamma_{1}^{2} \cos \gamma_{2}}{\gamma_{2}^{2}-\gamma_{1}^{2}}>n \pi \frac{\sin \theta}{\theta}>1,
$$

if $\beta-\alpha$ is sufficiently small, then

$$
g(1 ; \beta-\alpha)>1
$$

and we have

$$
f(1)<0 .
$$

Therefore, $\varphi(t)$ cannot be a characteristic function.

4. In this $\S$, we shall consider characteristic functions which satisfy (1.1). Assuming $\omega(t)$ in (1.2) to be a polynomial of $t$, from (1.3), $\omega(t)$ contains only terms of odd degree. Let

then

$$
\omega(t)=a_{1} t-a_{3} t^{3}+\cdots+(-1)^{n} a_{2 n+1} t^{2 n+1},
$$

that is

$$
\varphi(t)=(1+i \omega(t))^{-1}=\left[1+a_{1}(i t)+a_{3}(i t)^{3}+\cdots+a_{2 n+1}(i t)^{2 n+1}\right]^{-1},
$$

$$
\phi(t)=\frac{1}{Q(i t)}
$$


where $Q(z)$ is a polynomial of degree $2 n+1$ of real coefficients, without terms of $z^{2}, z^{4}, \cdots, z^{2 n}$,

(4.2) $\quad Q(z)=1+a_{1} z+a_{3} z^{3}+\cdots+a_{2 n+1} z^{2 n+1}$.

As (4.1) satisfies (1.1), we will consider if (4.1) be a characteristic function.

If $2 n+1$ roots $\alpha_{1}, \alpha_{2}, \cdots, \alpha_{2 n+1}$ of the polynomial $Q(z)$ are all real, then $\varphi(t)$ is rewritten

$$
\varphi(t)=\prod_{j=1}^{2 n+1}\left(1-\frac{i t}{\alpha_{j}}\right)^{-1},
$$

which proves that $\varphi(t)$ is a characteristic function, for the product of characteristic functions is a characteristic function. Conversely, in order that (4.3) satisfies (1.1), it is necessary that $\alpha_{1}, \alpha_{2}, \cdots, \alpha_{2 n+1}$ are roots of a polynomial of the form (4.2). We have proved the following

THEOREM 4.1. For any $n$ real numbers $\alpha_{1}, \alpha_{2}, \cdots, \alpha_{n}$ such that $\alpha_{j} \neq 0$ $(j=1, \cdots, n)$, in order that the characteristic function

$$
\phi(t)=\prod_{j=1}^{n}\left(1-\frac{i t}{\alpha_{j}}\right)^{-1}
$$

satisfies (1.1), it is necessary and sufficient that $n$ is odd and all the elementary symmetric functions of even degree of $\alpha_{1}, \alpha_{2}, \cdots, \alpha_{n}$ vanish.

It seems to be interesting, if there is a characteristic function (4.1) such that (4.2) has complex roots. If $n \leqq 2$, however, there is no such characteristic functions. Indeed, we have the following

THEOREM 4.2. In order that

$$
\varphi(t)=\left[1+a(i t)+b(i t)^{3}+c(i t)^{5}\right]^{-1}
$$

where $a, b, c$ are real numbers, be a characteristic function, it is necessary and sufficient that the polynomial

has no complex roots.

$$
Q(z)=1+a z+b z^{3}+c z^{5}
$$

Proof. Sufficiency : evident. Necessity: we shall prove that $\varphi(t)$ cannot be characteristic functions if $Q(z)$ has complex roots. From Theorem 2.1 and its Corollary, it is sufficient to prove in case when $c \neq 0$ and $Q(z)$ has three real roots and a pair of complex roots which are not pure imaginary. If $\phi(t)$ is a characteristic function, $a$ being real number, $\varphi(a t)$ is also a characteristic function. This shows that we can suppose that $c=1 . Q(z)$ has then the following form

$$
Q(z)=(z-\alpha)(z-\beta)(z-\gamma)\left\{z^{2}+(\alpha+\beta+\gamma) z-(\alpha \beta \gamma)^{-1}\right\} .
$$

Write

$$
s_{1}=\alpha+\beta+\gamma, \quad s_{2}=\alpha \beta+\alpha \gamma+\beta \gamma, \quad s_{3}=\alpha \beta \gamma .
$$

$\alpha, \beta$ and $\gamma$ must satisfy the following conditions

$$
\alpha \beta \gamma(\alpha+\beta+\gamma) \neq 0, \quad \alpha, \beta, \gamma \text { are real, }
$$




$$
\begin{aligned}
& s_{1}=s_{3}\left(s_{3}-s_{1} s_{2}\right), \\
& s_{1}^{2}<-4 s_{3}^{-1} .
\end{aligned}
$$

(4.7) is derived from the condition that the coefficient of $z^{2}$ vanishes. From (4.8), we have $s_{5}=\alpha \beta_{\gamma}<0$. We may suppose that $\alpha \leqq \beta \leqq \gamma$.

(a) Case when $\alpha \leqq \beta \leqq \gamma<0$. The real part $-(\alpha+\beta+\gamma) / 2$ of complex roots is positive. $Q(z)$ does not satisfy (3.4).

(b) Case when

$$
\alpha<0<\beta \leqq \gamma
$$

In this case we have

$$
s_{1}>0 \text {. }
$$

In fact, if $\alpha+\beta \geqq 0$ then $s_{1}=\alpha+\beta+\gamma>0$, and if $\alpha+\beta<0$ then $s_{2}=$ $(\alpha+\beta) \gamma+\alpha \beta<0, s_{3}<0$ and so $s_{1}=s_{3}^{2} /\left(1+s_{2} s_{3}\right)>0$. From (4.7) $\times(4.8)$, we have

$$
s_{1}^{3}<-4\left(s_{3}-s_{1} s_{2}\right)
$$

From (4.9),(4.10) and (4.11), we will prove that

$$
\alpha<-(\alpha+\beta+\gamma) / 2<0
$$

which completes the proof. These relations are invariant when we devide $\alpha, \beta$ and $\gamma$ by a same positive number, hence we can suppose that

$$
s_{1}=\alpha+\beta+\gamma=1
$$

Substituting 1 for $s_{1}$ in (4.11)

We have

$$
1<-4\left(s_{3}-s_{2}\right)
$$

$$
\begin{array}{rlrl} 
& & 1 / 4<s_{2}-s_{3} & =\alpha(\beta+\gamma)+\beta \gamma-\alpha \beta \gamma \\
& =\alpha(1-\alpha)+\beta \gamma(1-\alpha) \\
& & \leqq \alpha(1-\alpha)+(1-\alpha)^{3} / 4 \\
& & =(1 / 4)\left(1+\alpha-\alpha^{2}-\alpha^{3}\right) . \\
\therefore & \alpha^{3}+\alpha^{2}-\alpha<0, \\
\therefore & \alpha^{2}+\alpha-1>0 .
\end{array}
$$

$\alpha$ being negative, we have

$$
\alpha<-\frac{1}{2}=-\frac{\alpha+\beta+\gamma}{2}<0 .
$$

Since (3.4) is not satisfied, $\varphi(t)$ cannot be a characteristic function.

\section{COROLLARY. In order that}

$$
\varphi(t)=\left[1+a(i t)+b(i t)^{3}\right]^{-1}, \quad(a, b \text { real }, \quad b \neq 0)
$$

is a characteristic function, it is necessary and sufficient that there exists a real number $\alpha$ such that

$$
\begin{aligned}
& \frac{a}{\sqrt[3]{b}}=\frac{1}{\alpha}-\alpha^{2} \\
& \alpha \geqq \sqrt[3]{4} \text { or } \alpha<0
\end{aligned}
$$

If there is such $\alpha, \varphi(t)$ satisfies (1.1). 


\section{REFERENCES}

[ 1] H. CRAMÉr, Mathematical Methods of Statistics, 1946.

[2] PAUL LÉVY, Processus stochastiques et mouvement brownien, 1948. P. 186.

[3] EUGENE LUKACS and OTTO SZÁsz, Certain Fourier Transforms of distributions .

This appeared in the Canadian Journal of Mathematics, Vol. III, No. 2, 1951, after I had written this note.

THE INSititute of STATisTical Mathem atics

Soshigaya, Setagayaku, Tokyo 